\title{
Intra-osseous ultrasound for pedicle screw positioning in the subaxial cervical spine: an experimental study
}

\author{
Sven Rainer Kantelhardt • Hans Christoph Bock • Laila Siam • Jörg Larsen • \\ Ralf Burger • Wolfgang Schillinger • Volker Bockermann • Veit Rohde • Alf Giese
}

Received: 15 April 2009/Accepted: 18 June 2009 / Published online: 14 July 2009

(C) The Author(s) 2009. This article is published with open access at Springerlink.com

\begin{abstract}
Background In contrast to other regions of the human spine, dorsal fixation with rods and pedicle screws is comparatively rarely performed in the cervical spine. Although this technique provides a higher mechanical strength than the more frequently used lateral mass screws, many surgeons fear the relatively high rate of misplacements. This higher incidence is mainly due to the complex vertebral anatomy in this spinal segment. For correct screw placement, the availability of an immediate and efficient intra-operative imaging tool to ascertain the accuracy of the pedicle screw hole position would be beneficial. We have previously investigated the usefulness of an intraspinal, specifically, intra-osseous ultrasound technique in the lumbar spine. In this study its accuracy as a means of controlling intrapedicular screw hole positioning has been evaluated in the cervical spine.
\end{abstract}

S. R. Kantelhardt $(\bowtie) \cdot$ H. C. Bock $\cdot$ L. Siam $\cdot$ R. Burger $\cdot$

V. Bockermann $\cdot$ V. Rohde $\cdot$ A. Giese $(\bowtie)$

Department of Neurosurgery,

Georg August University of Göttingen,

Robert-Koch-Strasse 40,

37075 Göttingen, Germany

e-mail: sven.kantelhardt@web.de

e-mail: alf.giese@med.uni-goettingen.de

J. Larsen

Department of Neuroradiology,

Georg August University of Göttingen,

Robert-Koch-Strasse 40,

37075 Göttingen, Germany

W. Schillinger

Department of Cardiology and Pneumology,

Georg August University of Göttingen,

Robert-Koch-Strasse 40,

37075 Göttingen, Germany
Methods An endovascular ultrasound transducer was used for the intra-luminal scanning of 54 pedicle screw holes in cadaveric human spine specimens. Twenty-three of these had been intentionally misplaced (cortex breached). The resulting image files were assessed by three investigators blinded to both the procedure and the corresponding CT findings.

Findings The investigators differentiated correctly between adequately and poorly placed pedicle screw holes in $96 \%$ of cases. False negatives and false positives both occurred in no more than $1.8 \%$ of cases.

Conclusions Intrapedicular ultrasonography of pedicle screw holes in the cervical spine is a promising technique for the intra-operative assessment of bore hole placement and may increase operative safety and postoperative outcome in posterior cervical fusion surgery.

Keywords Pedicle screw placement - Bone sonography Cervical spine $\cdot$ Spinal instability

\section{Introduction}

Lateral mass screw placement and wiring of spinous processes are the most commonly used procedures for posterior stabilization of the subaxial cervical spine today. Although transpedicular screw fixation has long been described for $\mathrm{C} 2$ to $\mathrm{C} 7$ levels [8], these procedures are rarely undertaken for the vertebra $\mathrm{C} 3$ to $\mathrm{C} 6$, which are usually stabilized by lateral mass fixation [5, 23]. The latter yields good clinical results in most cases; however, some authors have raised concerns as to whether this technique results in sufficient stability, specifically when two or three levels are to be stabilized together $[2,15]$. Consequently, a combination of both ventral and posterior stabilization is 
often preferred $[2,26]$, and adjecent segments are often included in the instrumentation [13, 21]. Transpedicular screw fixation, in contrast, provides higher mechanical stability $[13,21]$ and showed higher pull-out strength in biomechanical studies $(1,214-677 \mathrm{~N}$ for pedicle versus 355-332 $\mathrm{N}$ for lateral mass screws in the subaxial cervical spine) $[6,7]$ and less loosening in fatigue analyses [7, 14]. In cases of severe instability, such as in complex fractures of the cervical spine or in ankylosing spondylitis [4], high mechanical stability is crucial, and transpedicular screw fixation may be preferred. Likewise, in patients that have severe anesthesiological risk factors, single-time pedicle screw placement might be preferred to two-time anterior/ posterior instrumentation with lateral mass screws and plate [13]. The particular disadvantage of this approach, however, is a higher rate of complications [12]. The specific anatomy of the cervical spine with high intra- and interindividual variability in vertebral size and shape [10] and small pedicular diameters [6, 11, 13, 16-18, 22, 24, 25] is considered largely responsible. Many authors therefore refrain from using pedicle screws in the cervical spine and accept the lower stability of lateral mass screws or combine dorsal and ventral stabilization at the cost of two surgical procedures.

We have recently reported a novel technique for the intraoperative evaluation of pedicle screw hole trajectories in the lumbar spine. Intra-osseous ultrasound (IOUS) [11] allowed the detection of pedicle perforations prior to permanent screw and implant placement. In an experimental study, blinded triplicate analysis of 24 pedicles in postmortem lumbar spine showed correct interpretation of the integrity of pedicle screw holes in $99 \%$ of cases. No pedicle perforation was missed [11]. The intraoperative evaluation of this technique demonstrated that the actual procedure as well as the interpretation of the diagnostic images were straightforward and required no more than approximately 1 min per pedicle. The aim of this study was to preclinically evaluate this technique in the examination of pedicle screw holes in the cervical spine.

\section{Materials and methods}

The experimental evaluation of intra-osseous ultrasound was carried out on four formalin-fixed adult human cervical spine specimens (including Th1 vertebra) with varying degrees of degenerative disk and synovial joint disease.

A total of 54 pedicle screw holes with a diameter of $3 \mathrm{~mm}$ and an approximate length of $30 \mathrm{~mm}$ were placed through the pedicles and into vertebral bodies from $\mathrm{C} 2$ to Th1 levels using a pneumatic drill. Twenty-three pedicle screw holes were intentionally placed with a deviation from the optimal trajectory, breaching the pedicular cortical surfaces or penetrating into the bony interforaminal channel of a vertebral artery. All pedicle screw holes were flooded with physiological saline solution prior to imaging.

For ultrasonographic imaging, an In-Vision Gold ${ }^{\mathrm{TM}}$ Intravascular Ultrasound System [3, 22] was fitted with an Eagle Eye Gold ${ }^{\mathrm{TM}}$, single-use Intravascular Ultrasound Imaging Catheter (Volcano Therapeutics Inc., Rancho Cordova, CA), designed for diagnostic intravascular ultrasound applications in cardiology (Fig. 1). The system provides real-time, two-dimensional $360^{\circ}$ images at a rectangular plane, relative to the long-axis of the catheter (Fig. 1). For intraluminal ultrasound imaging of pedicle screw holes, the catheter was advanced to the end of the pedicle screw holes and then retracted, acquiring a stack of approximately 150 images for each pedicle. Image arrays were exported as DICOM image files.

The spine specimens were scanned using a conventional 16-slice CT (MSCT) scanner (Aquilion ${ }^{\circledR}$, Toshiba Medical Systems, Neuss, Germany). Images were derived from a 240 -mm field of view to yield sections of 1.0-mm thickness reconstructed at $0.79-\mathrm{mm}$ intervals using both the highest resolution (bone) algorithm and a soft tissue algorithm. Image post-processing was carried out on a standard medical workstation $\left(V_{i t r e a}{ }^{\circledR}\right.$ 2, ViTAL Images Europe, Den Haag, The Netherlands). Anatomical details and surgical artifacts were specifically visualized by dedicated multi-planar image reconstruction and image appearances
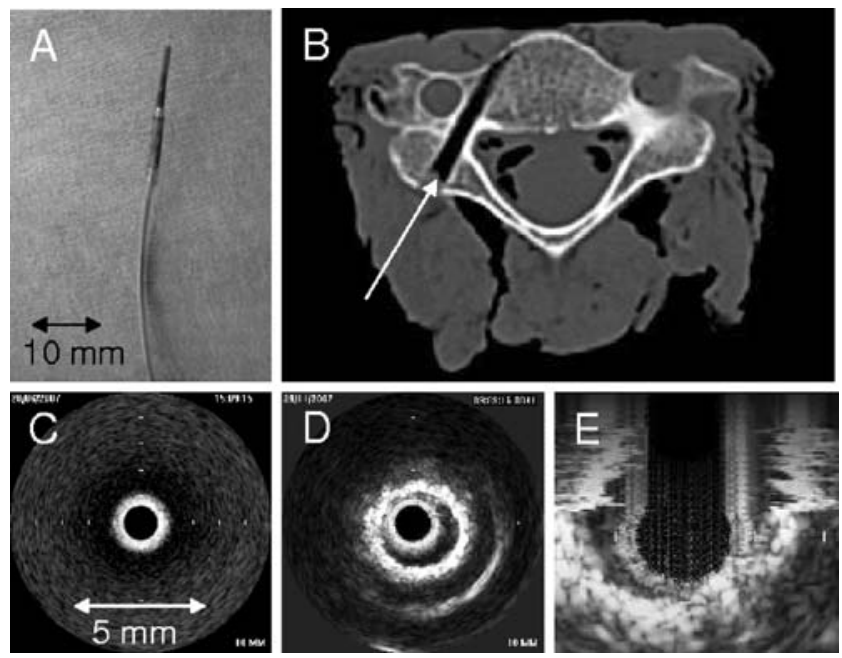

Fig. 1 a Intravascular ultrasound catheter (IVUS) as used for the intra-osseous ultrasound (IOUS) examinations in this study. b Transverse axial CT image showing the pediculation of an experimental vertebra (arrow). c and d IOUS images: c shows a $360^{\circ}$ ultrasound image in plain water, $\mathbf{d}$ image of the lumen of a pedicle screw hole of 3-mm diameter in cadaveric bone - the luminal surface of the pedicle screw hole appears as a high-intensity ring-like structure, enclosing the low-intensity signal of the lumen around the probe. e Shows a 3-dimensional reconstruction of an IOUS image stack of an experimental pedicle screw hole in cadaveric bone 
further altered by adjusting window settings. CT scanning demonstrated correct placement of pedicle screw holes in 31 cases. In 23 cases, deviation from the optimal trajectory was confirmed. Among these, a perforation lateral to the pedicle was ascertained in 5 cases, 4 trajectories crossed an intervertebral (neural) foramen, and 14 pedicle screw holes opened or crossed the channel of a vertebral artery.

The 54 ultrasound data arrays were independently reviewed by three investigators who were blinded to both the placement of pedicle screw holes and the corresponding CT findings. Following an introduction to the technique and the two principal appearances, investigators were asked to score the presence of cortical breaches as absent (0) or present (1). One investigator had no previous experience with intra-osseous ultrasonography. A non-parametric analysis of the scoring results was then carried out by performing a $\chi^{2}$ test. The significance level for testing was set at $95 \%$ (p-value $=0.05$ threshold).

In one patient with posttraumatic cervico-thoracic instability, we examined four pedicles (C7 and Th1) intraoperatively during dorsal cervico-thoracic spondylodesis: 3-mm holes were drilled transcutaneously. Drilling was performed using a pneumatic drill under robot assistance (SpineAssist $^{\mathrm{TM}}$, Mazor Surgical Technologies Inc., Ceasarea, Israel). Hereby, the robot guide arm is automatically positioned at the correct location according to the preoperative plan and serves as a guiding tool. Intraluminal ultrasound examination of the pedicle screw holes and pedicle screw placement were likewise performed via the robot guide arm and under fluoroscopic control. Postoperatively, CT scanning was carried out to confirm the result.

\section{Results}

Similar to the lumbar spine [11], intra-osseous ultrasound showed the lumen of pedicle screw holes as a low-intensity zone around the transducer. The luminal surface of pedicle screw holes appeared as a high-intensity ring-like structure at a distance (Fig. 2). Scanning of the 54 experimental pedicle screw holes for correct trajectories typically showed the entering of the probe into the pedicle screw hole at the level of the dorsal spinal lamina, which appeared as a highly intense linear or curved reflection (Fig. 2). The inner surfaces of the holes were also very highly reflective and depicted as a bright white ring with a smooth margin towards the hypoechoic artificial pedicular lumen. In cases of breaches of the pedicular cortex, ultrasonography showed a loss of the circular shape of the high-intensity bone signal at the perforation site. The low-intensity signal
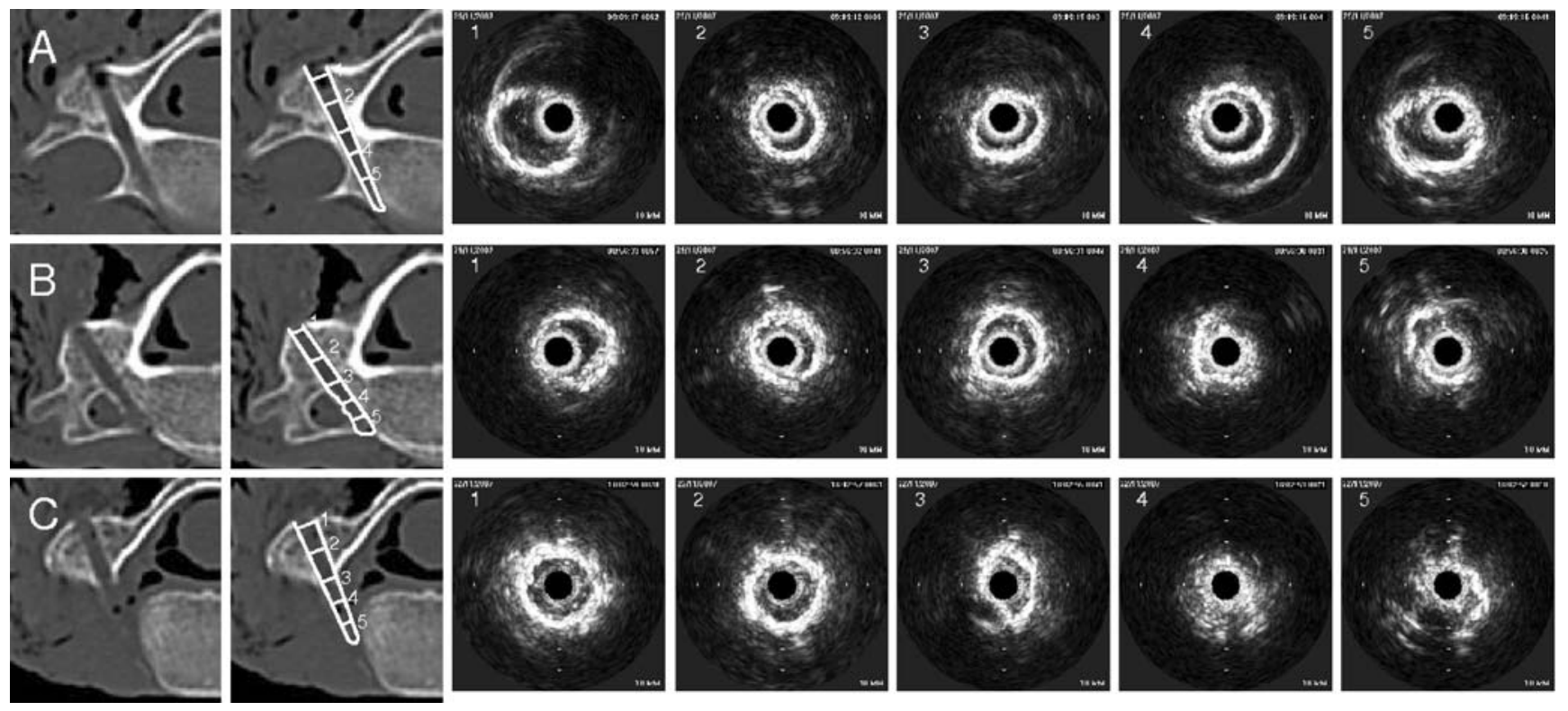

Fig. 2 CT scans and corresponding IOUS images of experimental pedicle screw holes in the cervical spine. The numbers on the CT images in the second column show the position in which the ultrasound probe was situated when the IOUS images with the corresponding number on the right hand side were taken. a Shows a correctly placed trajectory. All intra-osseous images (right, 1-5) show an intact ring-like structure, representing the luminal surface of the pedicle screw hole. b Shows a slightly deviated pedicle screw hole. Note the deformed luminal echo in 4 and the partial loss of the highly intense ring-like structure in 5 , corresponding to the perforation into the bony path of the vertebral artery. c Shows a completely deviated pedicle screw hole. While IOUS images 1 and 2 show regular appearances of an intra-osseous pedicle screw hole, a deformed signal is already seen in 3, while 4 and 5 show complete loss of the circular shape of the high-intensity bone signal and replacement of the lowintensity signal of the lumen of the pedicle screw hole by soft tissue reflectivity close to the probe 
of the screw hole lumen was replaced by soft tissue-like reflectivity close to the probe (Fig. 2c, positions 4 and 5).

If a vertebral artery channel was only just breached, the ring-shaped reflection of the bone vanished only partially or appeared dented (Fig. 2b, positions 4 and 5).

The analysis of the scoring results showed a high sensitivity of the technique: No significant interinvestigator variability could be noted, despite their different personal experience with IOUS. Two of the investigators (the most experiented and the one without any prior experience) made only one mistake each (53/54 correct, one false positive; Fig. 3), while one misinterpreted four ultrasound image stacks (50/54 correct, one false positive, three false negatives). Ninety-six percent of the pedicle examinations were thus interpreted correctly. Falsenegative and false-positive interpretations both occurred in $1.8 \%$ of the cases.

With respect to the intraoperative scanning of transcutaneously placed pedicle screw holes in one patient undergoing transcutaneous robot-assisted cervico-thoracic stabilization surgery, no substantial difference in the imaging appearances of pedicle screw holes in vivo as compared to cadaveric bone could be noted (Fig. 4), as previously shown in our studies in the lumbar spine [11]. The correct placement of pedicle screw holes, as suggested by intra-osseous sonography, could be confirmed by postoperative CT scanning.

Performance of the ultrasound studies required approximately $1 \mathrm{~min}$ for each pedicle screw hole. The combination of intra-osseous ultrasound and robot- assistance, as well as the transcutaneous application of the ultrasound probe via a guiding tube, presented no problems.

\section{Discussion}

In contrast to our previous study concerning the lumbar spine [11], pedicle screw holes in post-mortem cervical spines were drilled using a 3-mm pneumatic drill since this represents the most commonly used intraoperative technique for cervical pedicular screw placement in most institutions. IOUS examination of these pedicle screw holes resulted in perfectly round echoes from their bony margins. This simplified the interpretation of IOUS images as compared to those obtained in the lumbar spine where a surgical awl and pricker were used. Furthermore, the fastrotating drill left less bone debris in the pedicle screw holes, resulting in a clearer ultrasonic view.

It has to be mentioned that the smaller diameter of the pedicles in the cervical spine might also impair IOUS control of redirected pedicle screw holes. This is because the old and the redirected screw holes are more likely to cross each other in smaller pedicles than in bigger. However, IOUS might still show the reentering of the screw hole into the vertebral body. Furthermore, it can prove that a redirected pedicle screw hole does not breach the pedicle wall at the oposite side, because the characteristic ultrasound signal will be lost at the very site of the breach in the pedicle wall only, not on the other/opposite side.

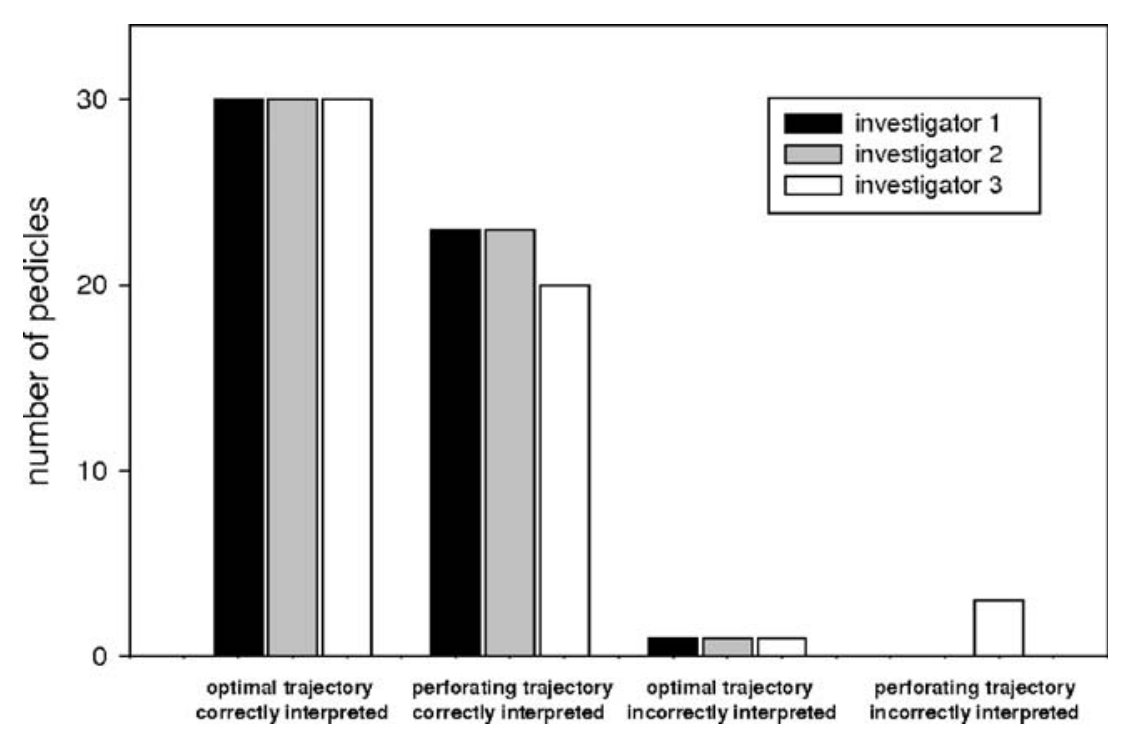

Fig. 3 Graphical presentation of the analysis of 54 ultrasound image stacks and CT scans of experimental pedicle screw holes. CT scanning confirmed correct trajectories of pedicle screw holes in 31 pedicles; 23 perforations were found. All three investigators misinterpreted one correctly placed pedicle screw hole as deviated (false positive), while one of the three investigators additionally misinterpreted three incorrectly placed pedicle screw holes (false negatives). On average, IOUS image stacks of experimentally placed pedicle screw holes in the cervical spine were correctly interpreted in $96 \%$ of cases, and false-positive and false-negative results occurred in $1.8 \%$ each 

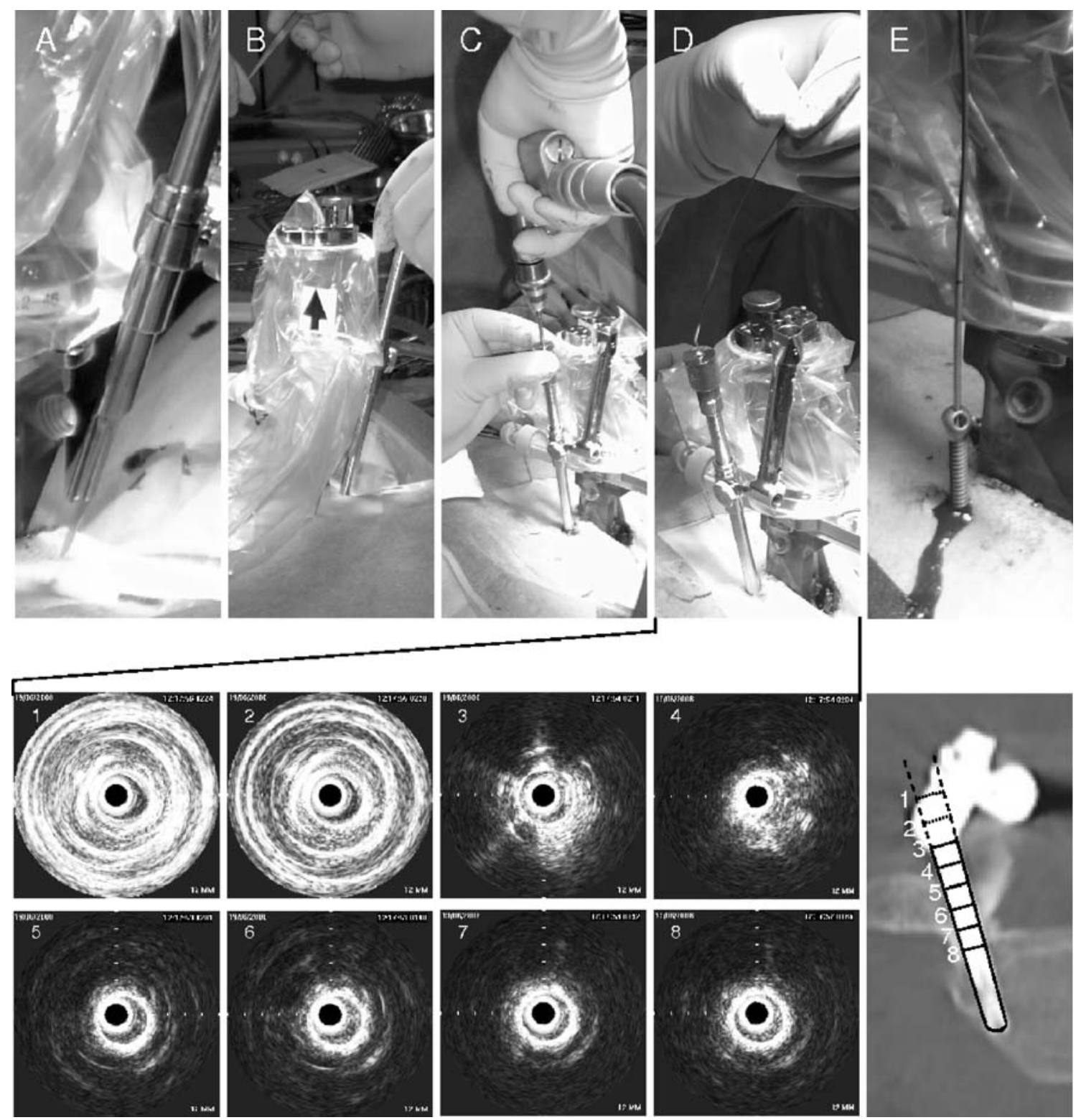

Fig. 4 Robot-assisted transcutaneous implantation of a pedicle screw in $\mathrm{C} 7$ vertebra. The upper panel shows the different steps of the implantation, skin incision (a), preparation of a transmuscular channel (b), drilling (c), IOUS examination via the drill guide (d) and screw implantation (e). The lower panel shows a series of IOUS images taken during the examination (d) with the corresponding positions of the IOUS probe marked as $1-8$ on the axial postoperative CT scan (on the very right). IOUS images 1 and 2 were taken when the ultrasound probe remained in the drill guide outside the pedicle (note the strong reflection at the metal surface), 3 and 4 were taken when the ultrasound probe left the drill guide but did not yet enter the bone, and 5-8 show the intact bony margin of the pedicle screw hole
On average, intra-osseous ultrasound stacks in this study were interpreted correctly in $96 \%$ of cases. This high level was achieved even by inexperienced investigators, although the number of researchers that have already applied IOUS is far too small to give reliable data concerning the learning curve for this technique.

For this study we used a simple intravascular ultrasound catheter designed for cardiological applications, i.e., not yet adapted for intra-osseous use. We have previously shown in a study in the lumbar spine that experimental IOUS images are directly comparable to intraoperative findings [11]. A specific adaptation of the IOUS device for intra-osseous use in combination with the advantages of an intraoperative application in comparison to the restrictions of our study design (in future, intraoperative scans may be repeated at will if their results are unclear, and IOUS images may also be correlated with other techniques, such as 2D-fluoroscopy or palpation of the pedicle screw hole walls with a curved probe, etc.) will further raise the rate of correct interpretations. 
While Abumi et al. report no more than 6.7\% (45/669) pedicle perforations in a retrospective series of cervical screw placements in open procedures using 2D-fluoroscopic control [1] only, other authors have described significantly higher rates for this technique. Ludwig et al., for example, report on a cadaveric study with $87.5 \%$ pedicle perforations when screws were placed according to anatomical landmarks and still 55\% misplacements when pedicles were directly visualized by laminoforaminotomy [18]. Because of these rates of misplacement, many surgeons refrain from pedicle screw placement in the cervical spine $[6,12,15$, $17-19,23,25,26]$. By doing so, they do however accept a lower mechanical stability $[15,20]$ or the need for a second operation from a ventral approach. Other authors, like us, use image guidance to assist stabilization. By this means, Ludwig et al. could reduce the perforation rate in their experimental study to $24 \%$. Kamimura et al. also applied computer-assisted image guidance, resulting in a similar rate $(23 \%)$ of perforations, also in an experimental setting [9]. In their prospective study, Richter et al. report a rate of only $3 \%$ pedicle perforations under computerized image guidance (in comparison to $8.6 \%$ for conventional screw placement) [21], while Kast et al. report a rate of $51 \%(n=$ 19 patients of whom 18 were operated upon using assistance from computerized image guidance) [13].

These data show that, even when image-guidance is applied, a significant risk of pedicle perforation remains. It is probable that the rate may still be higher for transcutaneous procedures. Fortunately, the rate of neurological symptoms related directly to pedicular screw misplacement is actually much lower $(0.3 \%$ in Abumi's retrospective analysis of conventionally placed screws, $0 \%$ in Richter's and $7 \%$ in Kast's prospective study of computer-assisted screw placement) $[1,13,21]$. However, the rate of revisions required rises with the number of screws implanted per patient, since a single symptomatic misplacement already requires surgical revision, irrespective of the number of correctly placed screws. Furthermore, other complications that might be attributed to screw deviations, such as narrowing of the vertebral artery channel with a consequent reduction in cerebral blood flow through this vessel or reduced mechanical strength of the fixation, might cause further problems. A direct disruption or punctation of the vertebral artery, however, will not be avoided by IOUS. This complication would be directly visible by strong bleeding from the pedicle screw hole even without application of IOUS. Fortunately, this seems to be a rather rare complication as it has not been reported in the clinical studies mentioned above $[1,13,21]$. If the bony interforaminal channel of a vertebral artery is breached, without acute disruption of the artery wall, IOUS could help the surgeon to identify the breach and avoid compression of the artery by screw implants.
Considering the numbers of breached pedicles in the recent clinical studies, the rate of $96 \%$ correctly interpreted pedicular screw hole trajectories established by this study might be sufficient to reduce the number of screw deviations significantly. This would enable operating surgeons to use cervical pedicle screws more frequently and thus provide the benefit of better mechanical stability to their patients. Furthermore, this study has demonstrated that a combination of IOUS as a control technique and (transcutaneous) image-guided procedures for pedicular screw hole placement may easily be combined.

\section{Conclusions}

IOUS is a fast and efficient technique for the intraoperative control of pedicle screw hole placement in the cervical spine. It seems likely to reduce the rate of misplaced screws and would thus make cervical pedicle screw placement a safer and more stable alternative to lateral mass screw placement, and would further reduce the need for combined ventral/dorsal approaches to the cervical spine.

Acknowledgement The cervical spine specimens were kindly provided by the Anatomical Institute of the University of Göttingen, Germany.

Open Access This article is distributed under the terms of the Creative Commons Attribution Noncommercial License which permits any noncommercial use, distribution, and reproduction in any medium, provided the original author(s) and source are credited.

\section{References}

1. Abumi K, Shono Y, Ito M, Taneichi H, Kotani Y, Kaneda K (2000) Complications of pedicle screw fixation in reconstructive surgery of the cervical spine. Spine 25(8):962-969

2. Albert TJ, Vacarro A (1998) Postlaminectomy kyphosis. Spine 23 (24):2738-2745

3. Bocksch W, Wellnhofer E, Schartl M, Dreysse S, Klimek W, Franke R, Musci M, Hetzer R, Fleck E (2000) Reproducibility of serial intravascular ultrasound measurements in patients with angiographically silent coronary artery disease after heart transplantation. Coron Artery Dis 11(7):555-562

4. Einsiedel T, Schmelz A, Arand M, Wilke HJ, Gebhard F, Hartwig E, Kramer M, Neugebauer R, Kinzl L, Schultheiss M (2006) Injuries of the cervical spine in patients with ankylosing spondylitis: experience at two trauma centers. J Neurosurg Spine $5(1): 33-45$

5. Heller JG, Silcox DH 3rd, Sutterlin CE 3rd (1995) Complications of posterior cervical plating. Spine 20(22):2442-2448

6. Jones EL, Heller JG, Silcox DH, Hutton WC (1997) Cervical pedicle screws versus lateral mass screws. Anatomic feasibility and biomechanical comparison. Spine 22(9):977-982

7. Johnston TL, Karaikovic EE, Lautenschlager EP, Marcu D (2006) Cervical pedicle screws vs. lateral mass screws: uniplanar fatigue analysis and residual pullout strengths. Spine J 6(6):667672 
8. Judet J, Roy-Camille R, Zerah JC, Saillant G (1970) Fractures of the cervical spine: fracture-separation of the articular column. Rev Chir Orthop Reparatrice Appar Mot 56(2):155-164

9. Kamimura M, Ebara S, Itoh H, Tateiwa Y, Kinoshita T, Takaoka K (2000) Cervical pedicle screw insertion: assessment of safety and accuracy with computer-assisted image guidance. J Spinal Disord 13(3):218-224

10. Kantelhardt SR, Oberle J, Derakhshani S, Kast E (2005) The cervical spine and its relation to anterior plate-screw fixation: a quantitative study. Neurosurg Rev 28(4):308-312

11. Kantelhardt SR, Bock HC, Larsen J, Bockermann V, Schillinger W, Rohde V, Giese A (2009) Intra-osseous ultrasound in the placement of pedicle screws in lumbar spine. Spine 15;34(4):400407

12. Karaikovic EE, Daubs MD, Madsen RW, Gaines RW Jr (1997) Morphologic characteristics of human cervical pedicles. Spine 22 (5):493-500

13. Kast E, Mohr K, Richter HP, Börm W (2006) Complications of transpedicular screw fixation in the cervical spine. Eur Spine J 15 (3):327-334

14. Kothe R, Rüther W, Schneider E, Linke B (2004) Biomechanical analysis of transpedicular screw fixation in the subaxial cervical spine. Spine 1;29(17):1869-1875

15. Kowalski JM, Ludwig SC, Hutton WC, Heller JG (2000) Cervical spine pedicle screws: a biomechanical comparison of two insertion techniques. Spine 25(22):2865-2867

16. Levine AM, Mazel C, Roy-Camille R (1992) Management of fracture separations of the articular mass using posterior cervical plating. Spine 17(10 Suppl):S447-S454

17. Ludwig SC, Kowalski JM, Edwards CC 2nd, Heller JG (2000) Cervical pedicle screws: comparative accuracy of two insertion techniques. Spine 25(20):2675-2681

18. Ludwig SC, Kramer DL, Balderston RA, Vaccaro AR, Foley KF, Albert TJ (2000) Placement of pedicle screws in the human cadaveric cervical spine: comparative accuracy of three techniques. Spine 25(13):1655-1667

19. Panjabi MM, Shin EK, Chen NC, Wang JL (2000) Internal morphology of human cervical pedicles. Spine 25(10):11971205

20. Richter M, Wilke HJ, Kluger P, Neller S, Claes L, Puhl W (2000) Biomechanical evaluation of a new modular rod-screw implant system for posterior instrumentation of the occipito-cervical spine: in-vitro comparison with two established implant systems. Eur Spine J 9(5):417-425

21. Richter M, Cakir B, Schmidt R (2005) Cervical pedicle screws: conventional versus computer-assisted placement of cannulated screws. Spine 30(20):2280-2287

22. Rodriguez-Granillo GA, McFadden EP, Aoki J, van Mieghem CA, Regar E, Bruining N, Serruys PW (2006) In vivo variability in quantitative coronary ultrasound and tissue characterization measurements with mechanical and phased-array catheters. Int $\mathbf{J}$ Cardiovasc Imaging 22(1):47-53

23. Shin EK, Panjabi MM, Chen NC, Wang JL (2000) The anatomic variability of human cervical pedicles: considerations for transpedicular screw fixation in the middle and lower cervical spine. Eur Spine J 9(1):61-66

24. Swank ML, Sutterlin CE 3rd, Bossons CR, Dials BE (1997) Rigid internal fixation with lateral mass plates in multilevel anterior and posterior reconstruction of the cervical spine. Spine 22(3):274-282

25. Tanaka N, Fujimoto Y, An HS, Ikuta Y, Yasuda M (2000) The anatomic relation among the nerve roots, intervertebral foramina, and intervertebral discs of the cervical spine. Spine 25(3):286-291

26. Uğur HC, Attar A, Uz A, Tekdemir I, Egemen N, Cağlar S, Genç Y (2000) Surgical anatomic evaluation of the cervical pedicle and adjacent neural structures. Neurosurgery 47(5):1162-1168

\section{Comment}

The aim of the article is clear and the subject of clinical utility. From this early experience, essentially in cadavers, probing the pedicle with IOUS seems like a useful adjunct in trying to reduce misplacement of cervical pedicle screws. Because the size (width) of cervical pedicles is small, it may become difficult to obtain a second reliable ultrasound signal in a second pass following one where a misplaced trajectory (and therefore a bone breach) has been identified with IOUS. The authors also address this issue and seem to be confident that the use of IOUS will enable visualization of a second correctly positioned trajectory. The authors should be commended for their effort in trying to make this at times challenging surgical technique less prone to neurological and vascular morbidity and more efficacious from the biomechanical standpoint.

Manuel Cunha e Sa

Almada, Portugal 